\title{
Features of the Synchronization of Spiral Wave Structures in Interacting Lattices of Nonlocally Coupled Maps
}

\author{
A. V.Bukh, V.S. Anishchenko
}

The features of external and mutual synchronization of spiral wave structures including chimera states in the interacting two-dimensional lattices of nonlocally coupled Nekorkin maps are investigated. The cases of diffusive and inertial couplings between the lattices are considered. The lattices model a neuronal activity and represent two-dimensional lattices consisting of $N \times N$ elements with $N=200$. It is shown that the effect of complete synchronization is not achieved in the studied lattices, and only the regime of partial synchronization is realized regardless of the case of coupling between the lattices. It is important to note that the conclusion is applied not only to the regimes of spiral wave chimeras, but also to the regimes of regular spiral waves.

Keywords: synchronization, two-dimensional lattice, spiral wave, spiral wave chimera, inertial and diffusing coupling

Received March 30, 2020

Accepted May 18, 2020

The reported study was funded by RFBR and DFG according to the research project No. 20-52-12004. A. V. B. thanks for the financial support provided by RFBR, project number 19-32-90005.

Andrei V. Bukh

buh.andrey@yandex.ru

Vadim S. Anishchenko

wadim@info.sgu.ru

Saratov State University

ul. Astrakhanskaya 83, Saratov, 410010 Russia

RUSSIAN JOURNAL OF NONLINEAR DYNAMICS, 2020, 16(2), 243-257 


\section{Introduction}

Formation and evolution of various dissipative structures in complex ensembles of interacting oscillators represent one of the key research directions in modern natural science [1-9]. In the last 15 years, the attention of specialists in nonlinear dynamics and related scientific fields has been focused on the studies of so-called "chimera states" [10-13]. These states are characterized by the coexistence of clusters of oscillators with coherent (synchronous) and incoherent (asynchronous) dynamics in the ensemble space. Of particular interest is the synchronization of chimera states in multicomponent systems and networks. Recently, various synchronization scenarios in multiplex and multilayer networks were studied [14-22], such as generalized synchronization [23], interlayer (external and mutual) synchronization [24-29], relay (remote) synchronization [30, 31], and explosive synchronization [32-35]. Among a large variety of chimera states [26, 36-51, 53-64], here we concentrate on so-called "spiral wave chimera structures" which are observed in 2D ensembles of coupled nonlinear oscillators [13, 65-80]. These were typically found in the case of nonlocal coupling topology of network elements. These structures represent spiral waves which rotate around incoherent cores. The network elements in the regions of rotating spiral waves are characterized by coherent dynamics, while the elements inside the incoherent cores oscillate asynchronously. We note that spiral wave chimeras were observed in both numerical and experimental [71] studies of the dynamics of $2 \mathrm{D}$ ensembles whose individual oscillators are described, as a rule, by systems of ordinary differential equations. The model of a 2D lattice consisting of nonlocally coupled discrete-time oscillators which describe neural activity [81] has been first proposed and explored in [80]. It was shown that this discrete model of the 2D lattice can exhibit all typical spiral wave structures, including spiral wave chimeras, which were found earlier for networks of coupled continuous-time systems [13, 66-74, 78, 79]. Effects of the external and mutual synchronization of spiral wave structures including spiral wave chimera states are studied and described in detail. It is shown that incoherent cores of spiral wave chimera states do not realize a complete synchronization effect, but synchronize only partially even if the value of the interlattice coupling strength parameter of the interacting $2 \mathrm{D}$-ensembles is sufficiently large. One possible reason for that fact is the type of the coupling function. We use the diffusive coupling which is given by coordinate states before $[82,83]$. Here we use both diffusive and inertial coupling functions. The aim of the research is to explore similarities and differences in the synchronization effect for both cases. In particular, the question should be answered: is the complete synchronization of incoherent cores possible?

\section{Models and methods}

In this paper we consider the synchronization of spiral wave structures which are realized in the network of nonlocally coupled Nekorkin maps which model neuronal activity with different coupling functions between interacting ensembles.

\subsection{Two coupled 2D lattices of Nekorkin maps}

Two 2D lattices with $200 \times 200$ nonlocally coupled Nekorkin maps are able to demonstrate synchronization effects between spiral wave structures when the intralayer coupling is nonlocal. We assume the coupling between the lattices to be multiplex and symmetrical or asymmetrical. This means that only corresponding oscillators of the lattices are asymmetrically coupled via their coordinates. In this case the coupled lattices are described by the following system 
of equations:

$$
\begin{aligned}
x_{i, j}^{t+1} & =f\left(x_{i, j}^{t}, y_{i, j}^{t}\right)+\sum_{m_{x}, n_{x}} G_{x}\left(x_{m_{x}, n_{x}}^{t}, x_{i, j}^{t}\right)+\Gamma_{u x}\left(u_{i, j}^{t}, x_{i, j}^{t}\right), \\
y_{i, j}^{t+1} & =g\left(x_{i, j}^{t}, y_{i, j}^{t}\right), \\
u_{i, j}^{t+1} & =f\left(u_{i, j}^{t}, v_{i, j}^{t}\right)+\sum_{m_{u}, n_{u}} G_{u}\left(u_{m_{u}, n_{u}}^{t}, u_{i, j}^{t}\right)+\Gamma_{x u}\left(x_{i, j}^{t}, u_{i, j}^{t}\right), \\
v_{i, j}^{t+1} & =g\left(u_{i, j}^{t}, v_{i, j}^{t}\right),
\end{aligned}
$$

where variables $x_{i, j}^{t}, y_{i, j}^{t}$ define the dynamics of the oscillators in the first lattice and variables $u_{i, j}^{t}, v_{i, j}^{t}$ determine the dynamics of the oscillators in the second lattice. The functions

$$
\begin{aligned}
& f(x, y)=x+x(x-a)(1-x)-y-\beta H(x-d), \\
& g(x, y)=y+\varepsilon(x-J)
\end{aligned}
$$

determine the dynamics of the single Nekorkin map [81]. Here $H(x)$ is the Heaviside step function. The intralayer coupling functions are described as follows:

$$
\begin{aligned}
& G_{x}\left(x_{k, l}, x_{i, j}\right)=\left(f\left(x_{k, l}, y_{k, l}\right)-f\left(x_{i, j}, y_{i, j}\right)\right)\left(\sigma_{x}\right) /\left(B_{i, j}^{x}\right), \\
& G_{u}\left(u_{k, l}, u_{i, j}\right)=\left(f\left(u_{k, l}, v_{k, l}\right)-f\left(u_{i, j}, v_{i, j}\right)\right)\left(\sigma_{u}\right) /\left(B_{i, j}^{u}\right) .
\end{aligned}
$$

The interlayer coupling functions in the case of diffusion coupling are given below:

$$
\begin{aligned}
& \Gamma_{u x}(u, x)=\gamma_{u x}(u-x), \\
& \Gamma_{x u}(x, u)=\gamma_{x u}(x-u) .
\end{aligned}
$$

In the case of inertial coupling between lattices we have the following:

$$
\begin{aligned}
& \Gamma_{u x}(u, x)=\gamma_{u x}(f(u, v)-f(x, y)), \\
& \Gamma_{x u}(x, u)=\gamma_{x u}(f(x, y)-f(u, v)),
\end{aligned}
$$

where $\gamma_{u x, x u}$ are the interlattice coupling strengths between corresponding oscillators of the first and second layer, respectively. In our studies we consider only the case of no-flux boundary conditions. The link indices $m_{x}, n_{x} \in \mathbb{N}$ are given as follows:

$$
\left\{\begin{array}{l}
\max \left(1, i-R_{x}\right) \leqslant m_{x} \leqslant \min \left(N, i+R_{x}\right), \\
\max \left(1, j-R_{x}\right) \leqslant n_{x} \leqslant \min \left(N, j+R_{x}\right)
\end{array}\right.
$$

for the first lattice, and indices $m_{u}, n_{u} \in \mathbb{N}$ in the second lattice are defined analogously by replacing $R_{x}$ with $R_{u}$. To account for potentially different coupling parameters in both networks, we introduce a subscript $x$ and $u$ for the first and second network in the coupling strengths $\sigma_{x}, \sigma_{u}$, coupling ranges $R_{x}, R_{u}$, numbers of nonlocal neighbors $B_{i, j}^{x}, B_{i, j}^{u}$ and neighbor indices $m_{x}, m_{u}, n_{x}, n_{u}$. The values of the coupling strengths are fixed as $\sigma_{x}=\sigma_{u}=0.08$.

In our studies we are interested in the dynamical regime of the spike oscillations in the Nekorkin map. This mode is exemplified in previous works $[82,83]$, where the phase portrait and time series for the variable $x^{t}$ are plotted, respectively. The map dynamics reflects a quasiperiodic mode in a lifted continuous-time system [84]. 


\subsection{Synchronization measurement}

The synchronization of oscillations between the lattices is quantified by calculating the number of synchronized elements $N_{s}$ in the lattices, which satisfy the condition $r_{i, j} \geqslant 0.95$, where the correlation coefficient $r_{i, j}$ between corresponding oscillators of the lattices is given as follows:

$$
\begin{aligned}
& r_{i, j}=\frac{\left\langle\tilde{x}_{i, j} \tilde{u}_{i, j}\right\rangle}{\sqrt{\left\langle\tilde{x}_{i, j}^{2}\right\rangle\left\langle\tilde{u}_{i, j}^{2}\right\rangle}}, \\
& \tilde{x}_{i, j}=x_{i, j}-\left\langle x_{i, j}\right\rangle, \quad \widetilde{u}_{i, j}=u_{i, j}-\left\langle u_{i, j}\right\rangle .
\end{aligned}
$$

The correlation coefficient $r_{i, j}$ is widely used when synchronization of coupled oscillators is studied [51, 52]. The corresponding oscillators are assumed to be synchronized if $r_{i, j} \geqslant 0.95$, otherwise they are desynchronized. The threshold value of $r_{i, j}^{\text {th }}=0.95$ is chosen because complete synchronization cannot be achieved in the case of parameter detuning in the interacting lattices. The condition $r_{i, j} \geqslant 0.95$ characterizes the maximum degree of synchronization of spatio-temporal structures which is possible in the regimes studied. Also, we use $N_{d}=N^{2}-N_{s}$, which denote the number of desynchronized oscillators.

\section{Numerical results for spiral wave structures}

Here we fix the parameters of single neurons $\alpha=0.2, \beta=0.04, J=0.15, \delta=0.5, \varepsilon=0.005$. We fix the intracoupling strength parameters $\sigma_{x}=\sigma_{u}=0.08$ as well. First, we obtain spiral wave structure with local coupling $\left(R_{x}=1\right)$ in the separate lattice (at $\gamma_{u x}=\gamma_{x u}=0$ ) using random initial values evenly distributed within corresponding intervals $\left(x_{i, j}^{0} \in[-0.4: 0.6]\right.$, $\left.y_{i, j}^{0} \in[-0.04: 0.06]\right)$. Then we vary the coupling range $R_{x}$ preserving the states of amplitudes $x_{i, j}, y_{i, j}$. We get spiral wave structures with different wavelengths as a result (Fig. 1). Figure 1 shows that the wavelength increases with increasing coupling range. Note that the incoherence area is not observed in the center of the spiral wave at small values of $R_{x}$ (Figs. 1a, 1b), but it rises in the center of the spiral wave when there is a sufficient increase in the value of the coupling range parameter (Fig. 1c). The interaction of the two coupled lattices with different given structures (Fig. 1) is the subject of this work.

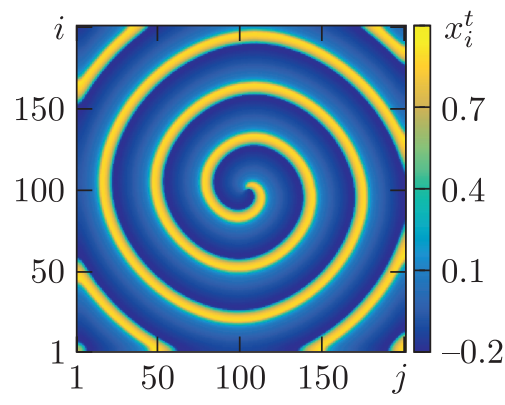

(a)

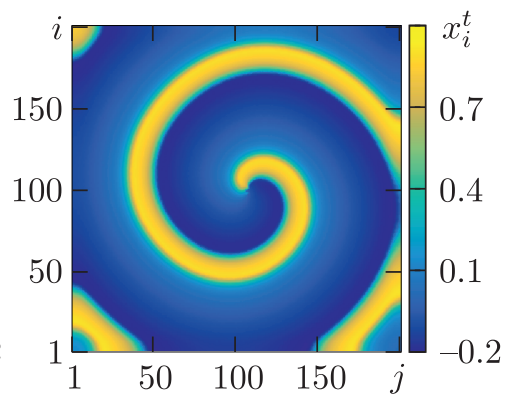

(b)

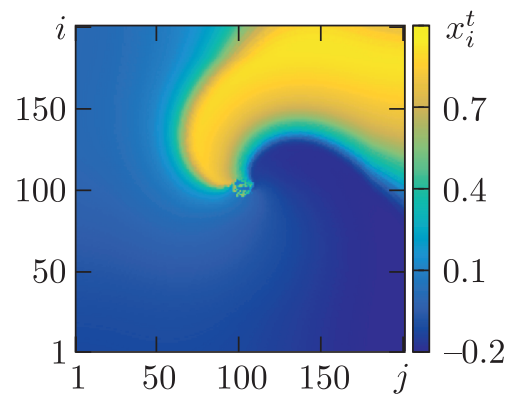

(c)

Fig. 1. Spiral wave structures in the single lattice of nonlocally coupled Nekorkin maps. Snapshots of $x_{i, j}$ in (2.1) for (a) $R_{x}=2$, (b) $R_{x}=5$, (c) $R_{x}=20$ with $\gamma_{u x}=\gamma_{x u}=0$. Other parameters: $\alpha=0.2$, $\beta=0.04, J=0.15, \delta=0.5, \varepsilon=0.005, \sigma_{x, u}=0.08$. 


\subsection{Interaction of spiral waves}

Now we consider the interaction of these two lattices with different ranges of the nonlocal coupling (2.1). This interaction was examined earlier for the case of diffusing coupling [82]. It was shown that both regular and spiral structures can be synchronized when the parameter of interlayer coupling strength increases. Here we introduce the inertial coupling and compare the results obtained with the ones known for the case of diffusive coupling. First, we choose regular spiral wave structures: the spiral wave at $R_{x}=2$ (Fig. 1a), and the spiral wave with $R_{u}=5$ (Fig. 1b). Coupling strength parameters are the same $\left(\gamma_{u x}=\gamma_{x u}=\gamma\right)$, which corresponds to mutual coupling between the lattices. Results on the interaction of these structures with the parameter of inertial coupling strength $\gamma=0.026$ are shown in Fig. 2 and show that there is synchronization of the lattices. Correlation coefficients $r_{i, j}<0.95$ when the interlayer coupling parameter $\gamma<0.025$, but almost all the oscillator pairs are synchronized for $\gamma \sim 0.026$. The resulting structure represents an intermediate case in comparison with initial structures in separate lattices (Fig. 2c). The described mechanism of the mutual synchronization repeats the one obtained for the diffusion coupling studied earlier. The important fact should be noted here. The regime of complete synchronization is not achieved even for regular spiral wave structures of Fig. 2. A small group of desynchronized oscillator pairs remains in the center of the lattices even for large values of the coupling strength parameter. We build the normalized dependence of the number of synchronized pairs $\left(\left\{N_{s}: r_{i, j}>0.95\right\}\right)$ on the interlayer strength parameter $\gamma$ in order to compare in detail the synchronization effect for both inertial and diffusive coupling cases (Fig. 3). The plot shows that the synchronization occurs at smaller values of the strength parameter $(\gamma \sim 0.024)$ for the diffusive coupling than for the inertial coupling $(\gamma \sim 0.026)$. And the number of desynchronized oscillator pairs is larger in the case of inertial coupling $\left(N_{d}=150\right.$ at $\gamma=0.25)$ than in the case of diffusive coupling $\left(N_{d}=115\right.$ at $\left.\gamma=0.25\right)$. In the described numerical experiment the lattices show qualitatively the same behavior for inertial and diffusive coupling between them.

Now we set unidirectional inertial coupling between lattices with structures at $R_{x}=2$ (Fig. 1a) and at $R_{u}=5$ (Fig. 1b). First, we consider the case when $\gamma_{u x}=0, \gamma_{x u}=\gamma$. The case corresponds to the impact of the structure in Fig. 1 on the structure in Fig. 1b. Then we increase the parameter $\gamma$, which controls the impact strength of the first (driver) lattice on the second (response) lattice. The results of numerical experiments, snapshots of the amplitudes

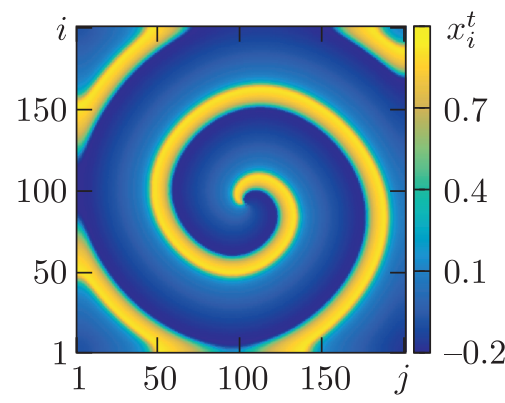

(a)

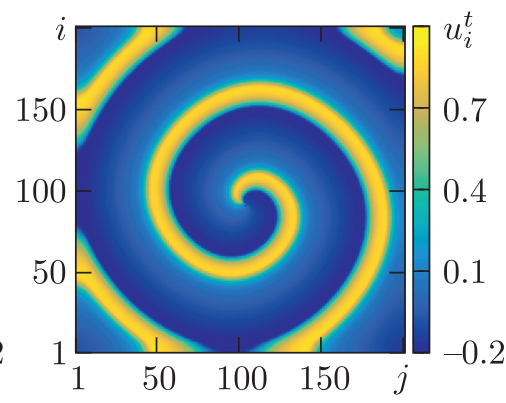

(b)

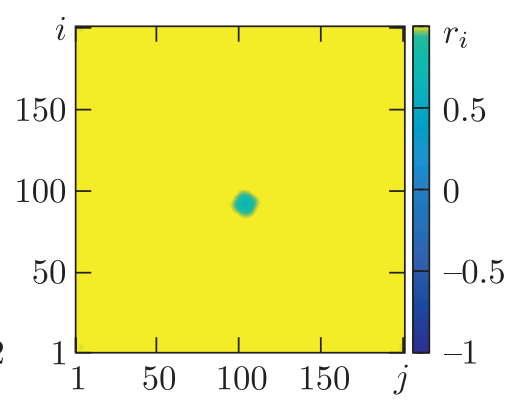

(c)

Fig. 2. Result of the mutual synchronization of spiral wave structures (fragments (a), (b) in Fig. 1) in the two inertially coupled lattices. Snapshots of $x_{i, j}$ ((a), first lattice with $\left.R_{x}=2\right)$ and $u_{i, j}$ ((b), second lattice with $R_{u}=5$ ) and correlation coefficients $r_{i, j}(\mathrm{c})$. Coupling strength $\gamma=0.026$. Other parameters: $\alpha=0.2, \beta=0.04, J=0.15, \delta=0.5, \varepsilon=0.005, \sigma_{x, u}=0.08$. 


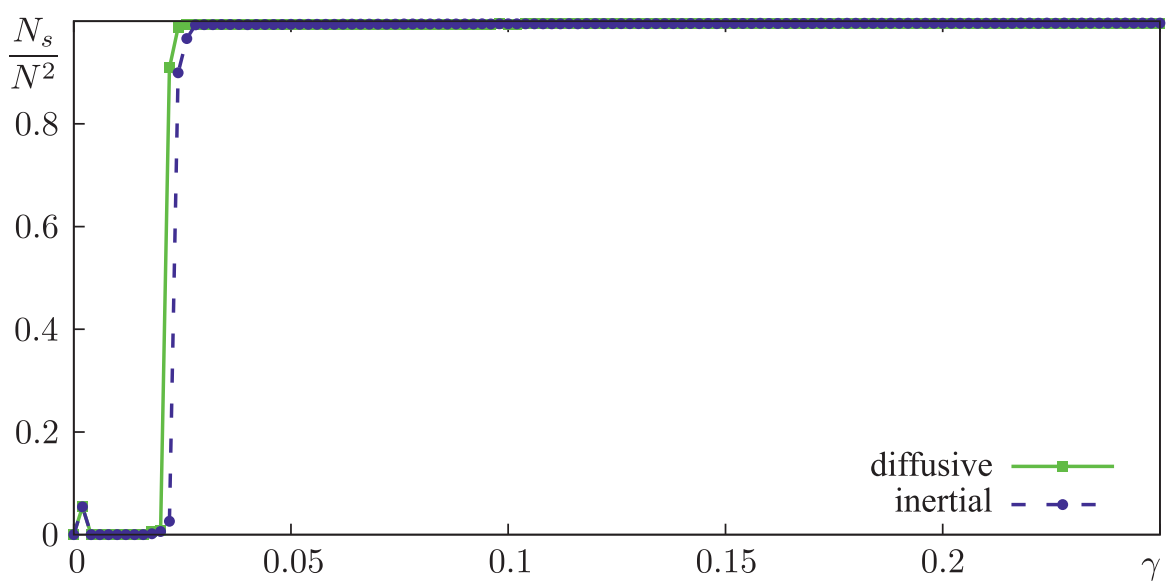

Fig. 3. Normalized number of synchronous oscillators $\left(\left\{N_{s}: r_{i, j}>0.95\right\}\right)$ versus the interlattice coupling strength $\gamma=\gamma_{u x}=\gamma_{x u}$, with $R_{x}=2, R_{u}=5$. Other parameters: $\alpha=0.2, \beta=0.04, J=0.15, \delta=0.5$, $\varepsilon=0.005, \sigma_{x, u}=0.08$.

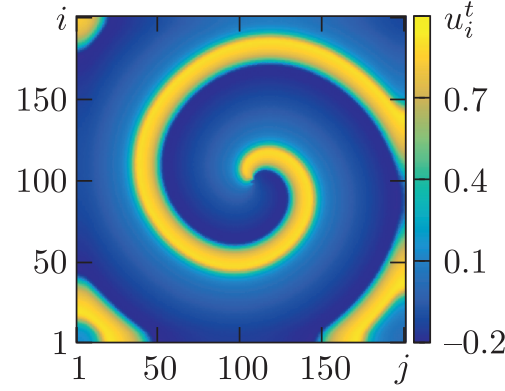

(a)

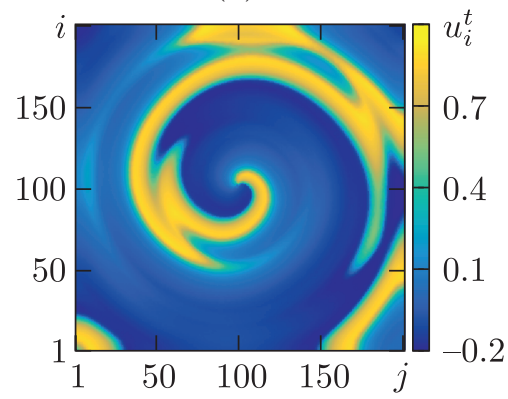

(b)

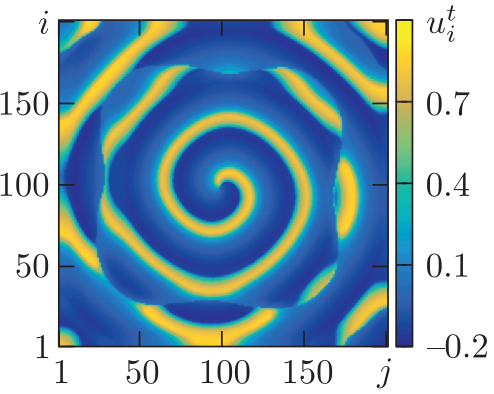

(c)

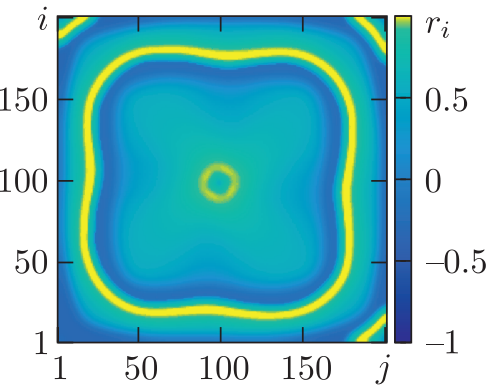

(d)

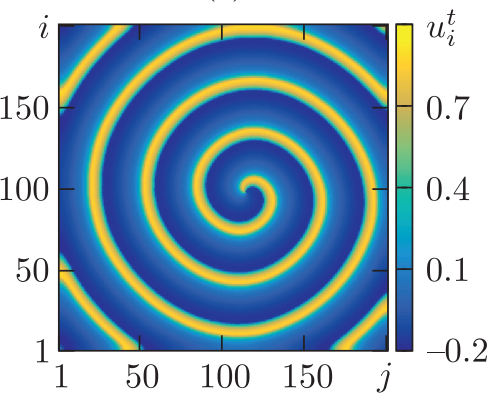

(e)

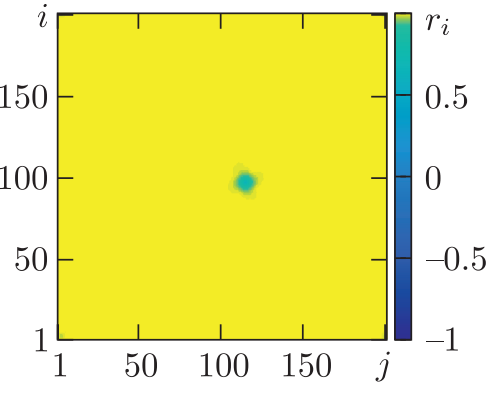

(f)

Fig. 4. Spiral wave structures in the response lattice at $R_{x}=2, R_{u}=5$. Snapshots of $u_{i, j}$ in $(2.1)$ with (a) $\gamma=0$, (b) $\gamma=0.012, N_{s}=3545$, (c) $\gamma=0.024, N_{s}=2254$, (e) $\gamma=0.168, N_{s}=36154$ with $\gamma_{u x}=0$, $\gamma_{x u}=\gamma$. Correlation coefficients with the first (driver) lattice for (d) $\gamma=0.024$, (f) $\gamma=0.168$. Other parameters: $\alpha=0.2, \beta=0.04, J=0.15, \delta=0.5, \varepsilon=0.005, \sigma_{x, u}=0.08$.

of the second (response) lattice are shown in Figs. 4a-4c, 4e). In the second lattice the spiral wave structure at $R_{u}=5$ is chosen with $\gamma=0$. Then a small part of the oscillators pairs is synchronized at $\gamma=0.012\left(N_{s}=3545\right)$, and the structure in the second (response) lattice is changed (Fig. 4b). The structure continues to alter and the number of synchronized pairs $N_{s}$ increases with the following increase in the parameter $\gamma(\gamma=0.024$ in Figs. 4c, 4d). At last, a large number of oscillator pairs are synchronized at $\gamma=0.168$ (Fig. 4f) and the response lattice 


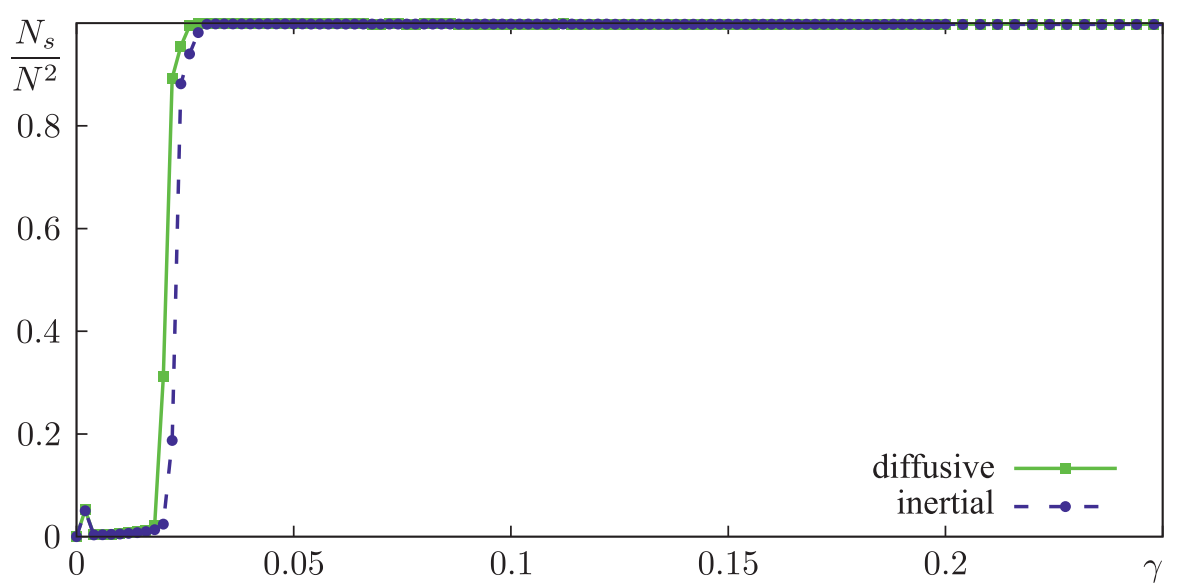

Fig. 5. Normalized number of synchronous oscillators $\left(\left\{N_{s}: r_{i, j}>0.95\right\}\right)$ versus the interlattice coupling strength $\gamma=\gamma_{u x}$, with $R_{x}=2, R_{u}=5$, and $\gamma_{x u}=0$. Other parameters: $\alpha=0.2, \beta=0.04, J=0.15$, $\delta=0.5, \varepsilon=0.005, \sigma_{x, u}=0.08$.

repeats the structure that is realized in the driver lattice (compare Fig. 1a and Fig. 4f). The results obtained for the case of diffusion coupling are similar to the ones presented in Fig. 4, but the synchronization occurs with larger values of $\gamma$ and a larger number of oscillators remain desynchronized. Now we change the direction of the coupling $\left(\gamma_{x u}=0, \gamma_{u x}=\gamma\right)$ and increase the value of the parameter $\gamma$. The structure in the second (driver) lattice with $R_{u}=5$ (Fig. 1b) impacts the structure in the first (response) lattice with $R_{x}=2$ (Fig. 1a). Results of numerical simulations (snapshots for the first lattice and correlation coefficients between lattices) show that at $\gamma=0.004$ the structure alters and a small part of oscillator pairs synchronize, and with $\gamma=0.028$ almost all the oscillators are synchronized. Thus, the change in the direction of the external coupling leads to the synchronization with smaller values of the parameter $\gamma$ and with a noticeably larger number of synchronized oscillator pairs. The results in the second case are qualitatively the same in the case of diffusion coupling. There is the only difference in the threshold value of parameter $\gamma$ at which the synchronization occurs (Fig. 5). This difference is the same for the mutual synchronization considered above.

The results described above show no qualitative differences in the behavior of lattices with diffusive and inertial couplings between them. The effect of external synchronization depends on the direction of the coupling. A large part of oscillators pairs remains desynchronized when the structure with the smaller wavelength impacts the structure with the larger wavelength. But almost all the oscillators are synchronized when the structure with the larger wavelength impacts the structure with the smaller wavelength. It may be assumed that the structure with the larger wavelength has a stronger effect than the structure with the smaller wavelength on the behavior of both lattices in the case of mutual synchronization.

\subsection{The interaction of the spiral wave and the spiral wave chimera}

Now we consider the interaction of two lattices (2.1) with the spiral wave at $R_{x}=5$ in the first lattice (Fig. 1b) and with the spiral wave chimera at $R_{x}=20$ in the second lattice (Fig. 1c). The intercoupling strengths are set the same $\left(\gamma_{u x}=\gamma_{x u}=\gamma\right)$, which corresponds to mutual coupling between the lattices. The results of the interaction of two lattices with increasing values of inertial interlattice coupling strength parameter are presented in Fig. 6. There is no 


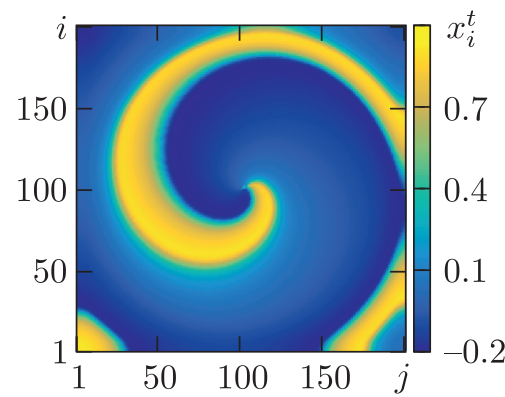

(a)

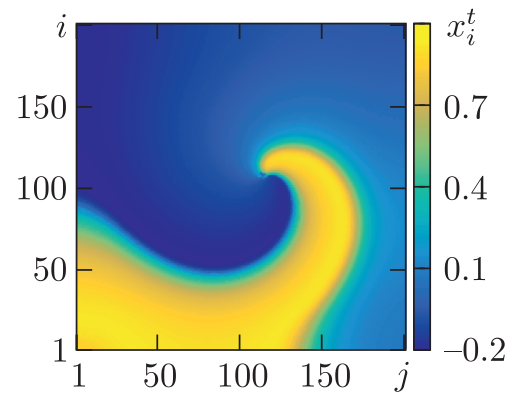

(d)

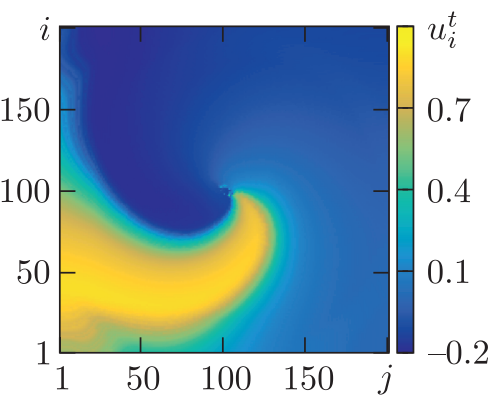

(b)

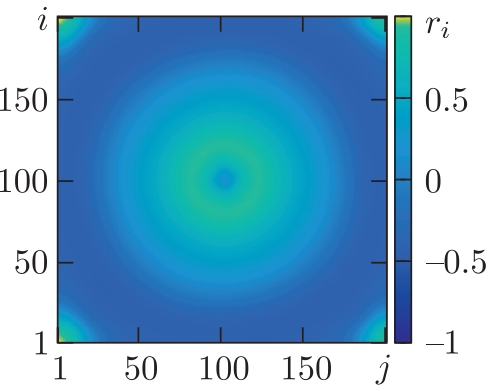

(c)

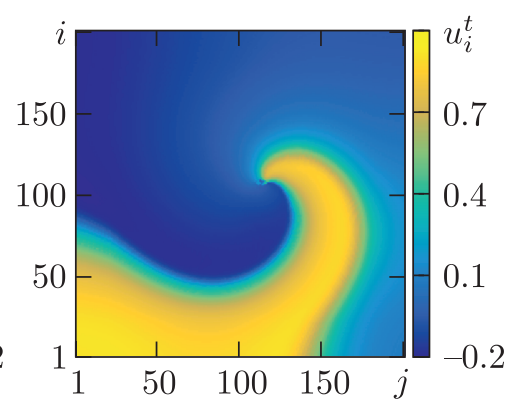

(e)

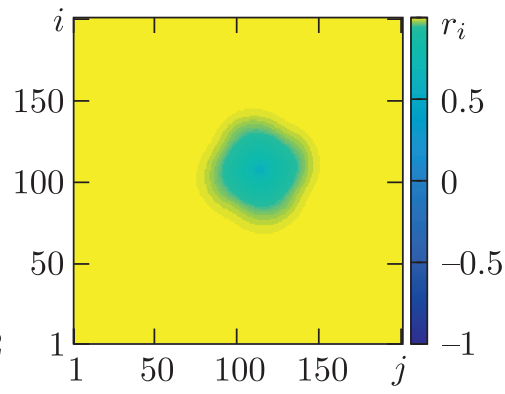

(f)

Fig. 6. Result of the mutual synchronization of spiral wave structures (fragments (a), (b) in Fig. 1) in the two inertially coupled lattices. Snapshots of $x_{i, j}\left((\mathrm{a})\right.$, (b), first lattice with $\left.R_{x}=5\right)$ and $u_{i, j}$ ((d), (e), second lattice with $\left.R_{u}=20\right)$ and correlation coefficients $r_{i, j}$ for the first lattice (c) and for the second lattice (f). Coupling strength $\gamma=0.004$ (a)-(c), and $\gamma=0.04$ (d)-(f). Other parameters: $\alpha=0.2, \beta=0.04, J=0.15, \delta=0.5, \varepsilon=0.005, \sigma_{x, u}=0.08$.

synchronization effect with $\gamma<0.015$, and the correlation coefficients $r_{i, j}<0.95$ (Figs. 6a-6c). But the synchronization of almost all the oscillator pairs appears for $\gamma \sim 0.04$. The structure obtained represents an intermediate case between the structures obtained in the separate lattice (compare Figs. 1b, 1c and Figs. 6d-6f). In the first lattice the wavelength increases with an increase in the value of $\gamma$ (Figs. 6a, 6d), and in the second lattice the incoherence area of the spiral wave chimera decreases (Figs. 6b, 6e). The results are similar for the case of diffusive coupling. The normalized dependence of the number of synchronized pairs $\left(\left\{N_{s}: r_{i, j}>0.95\right\}\right)$ on the interlayer strength parameter $\gamma$ (Fig. 7) shows that partial synchronization appears with smaller values of $\gamma \sim 0.024$ for the diffusive coupling than for the inertial coupling $(\gamma \sim 0.026)$, but the effect is qualitatively the same as in all the cases above.

The results presented in Fig. 8, a show that there is a cluster of desynchronized oscillators $(R \in[0.63: 0.66])$ near the excitation center of the regular spiral wave, when the incoherence cluster is not yet formed. This is a very important fact, which indicates that spiral wave chimera is formed long before the appearance of the obvious incoherence core.

In the case of the impact of the lattice with $R_{x}=2$ on the lattice with $R_{u}=5$, i.e., in the case of the impact of the structure with the smaller wavelength on the structure with the larger wavelength, the partial synchronization is realized only for sufficiently large values of the interlayer coupling strength as shown in the previous paragraph. But in the case of the impact of the lattice with $R_{x}=5$ on the lattice with $R_{u}=20$ there is no synchronization effect for either diffusive or inertial couplings. In this case, the maximal values of the correlation coefficients are close to $0.95\left(\max \left(r_{i, j}\right) \sim 0.9\right)$, and the structures are similar according to the 


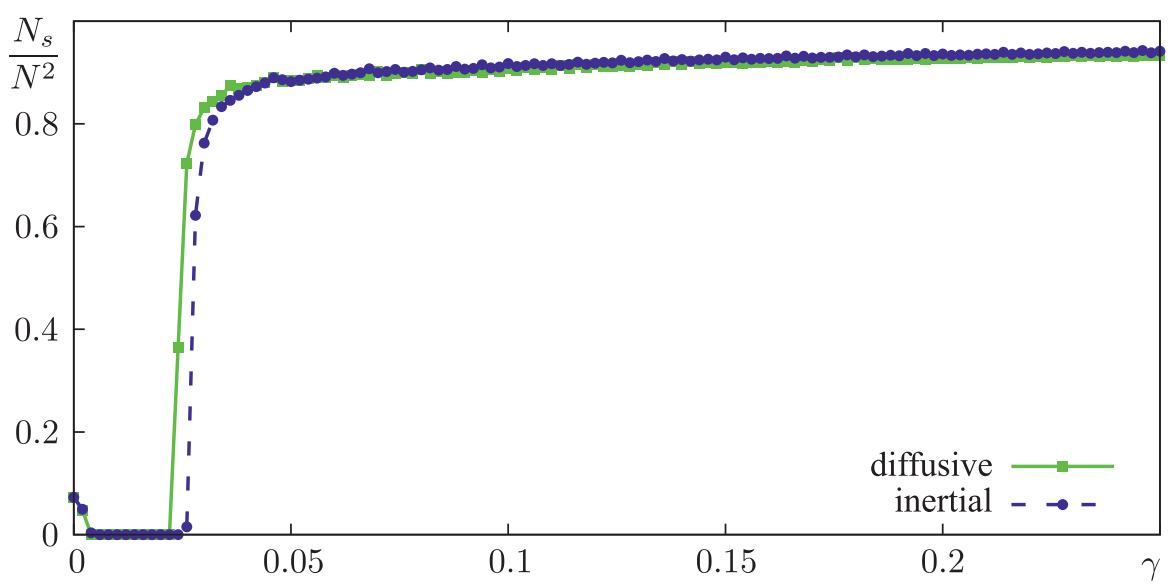

Fig. 7. Normalized number of synchronous oscillators $\left(\left\{N_{s}: r_{i, j}>0.95\right\}\right)$ versus the interlattice coupling strength $\gamma=\gamma_{u x}=\gamma_{x u}$, with $R_{x}=5, R_{u}=20$. Other parameters: $\alpha=0.2, \beta=0.04, J=0.15, \delta=0.5$, $\varepsilon=0.005, \sigma_{x, u}=0.08$.

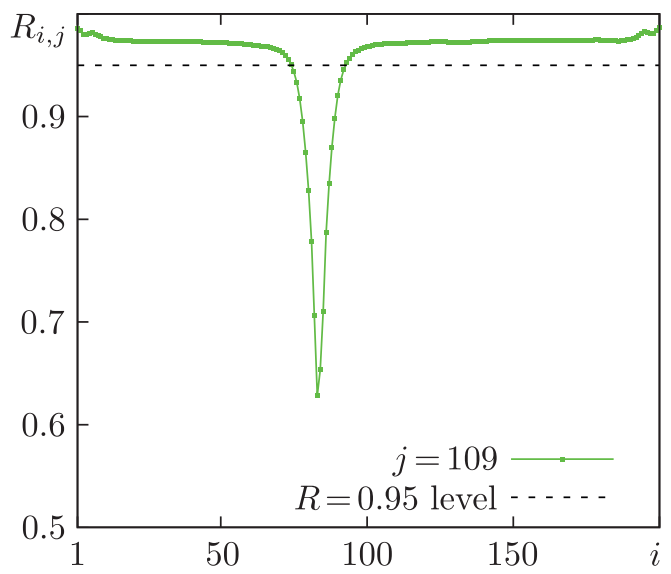

(a)

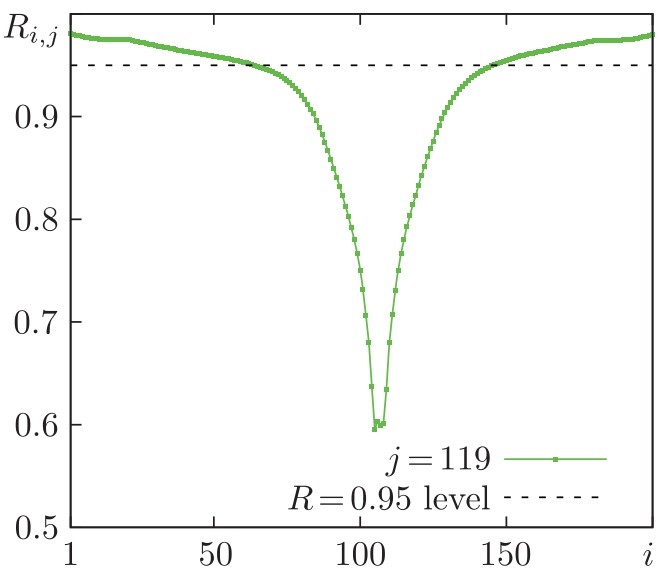

(b)

Fig. 8. Comparison of the synchronization of regular structures and the regular structures with chimera structures. 1D cuts of 2D maps of correlation coefficients with $\gamma=\gamma_{x u}=\gamma_{u x}=0.05$ and with (a) $R_{x}=2$, $R_{u}=5$; (b) $R_{x}=5, R_{u}=20$. Other parameters: $\alpha=0.2, \beta=0.04, J=0.15, \delta=0.5, \varepsilon=0.005$, $\sigma_{x, u}=0.08$.

snapshots of the second lattice for large values of $\gamma$. The results of the impact of the second lattice with $R_{u}=20$ on the first lattice with $R_{x}=5$ are presented in Fig. 9 and qualitatively correspond to the case of the impact of the lattice with $R_{u}=5$ on the lattice with $R_{x}=2$. The wavelength of the spiral wave increases with the increase in the $\gamma$ (Fig. 9a), and the first lattice repeats the structure of the second lattice at $\gamma \sim 0.038$ (Fig. 9b). The first lattice repeats the structure of the second lattice at $\gamma \sim 0.034$ in the case of diffusive coupling. The comparison of the dependence of the normalized number of synchronous oscillators on the interlattice coupling strength (Fig. 10) shows that the synchronization effect appears for smaller values of $\gamma$ in the case of the diffusive coupling than in the case of the inertial coupling as in all the cases above. There are no qualitative differences between lattices with diffusive and inertial couplings. The structure with the larger wavelength has a stronger effect on the structure with the smaller wavelength than the second on the first. 


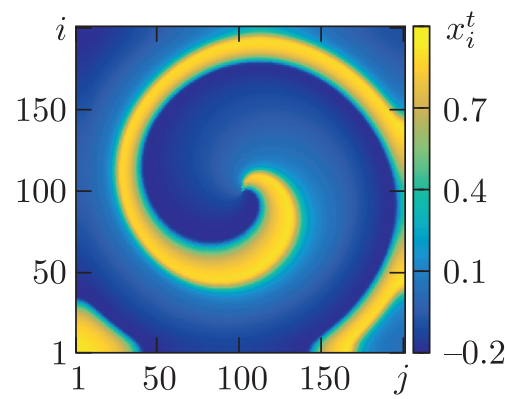

(a)

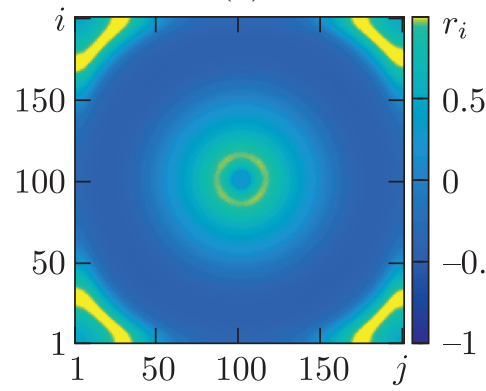

(c)

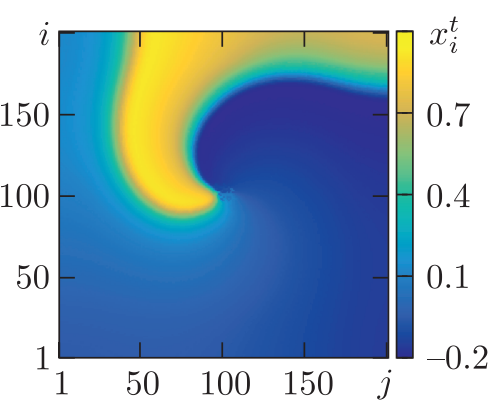

(b)

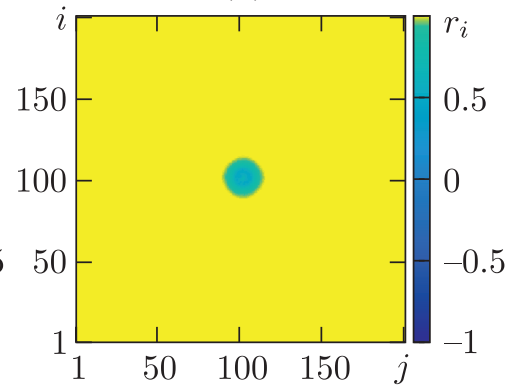

(d)

Fig. 9. Spiral wave structures in the response lattice at $R_{x}=5, R_{u}=20$. Snapshots of $x_{i, j}$ (a), (b) and correlation coefficients (c), (d) in (2.1) with (a), (c) $\gamma=0.004, N_{s}=1112$, (b), (d) $\gamma=0.038, N_{s}=39441$ with $\gamma_{x u}=0, \gamma_{u x}=\gamma$. Other parameters: $\alpha=0.2, \beta=0.04, J=0.15, \delta=0.5, \varepsilon=0.005, \sigma_{x, u}=0.08$.

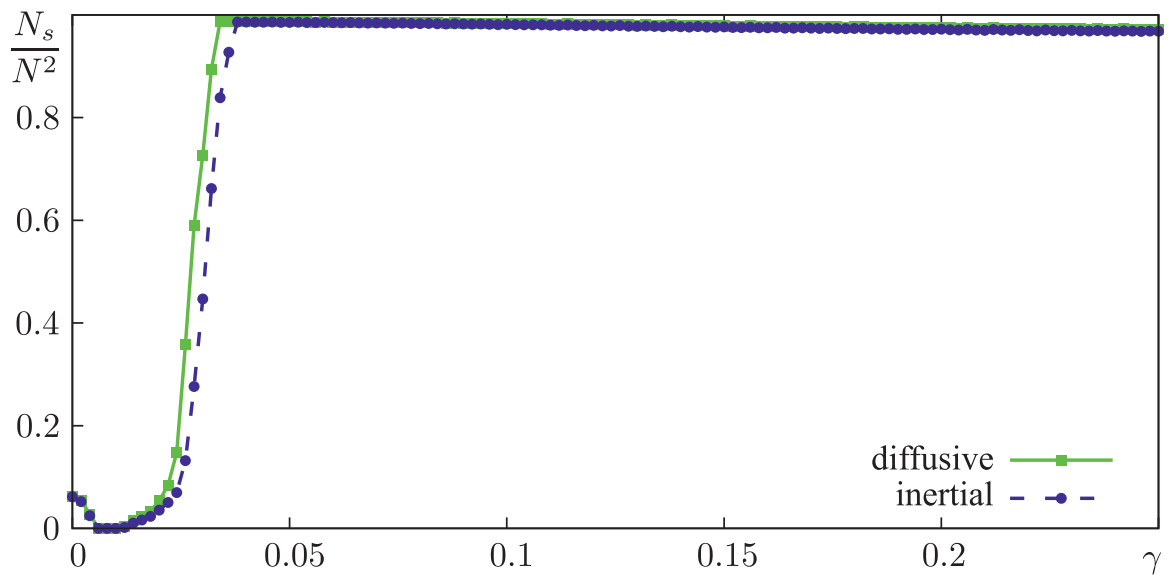

Fig. 10. Normalized number of synchronous oscillators $\left(\left\{N_{s}: r_{i, j}>0.95\right\}\right)$ versus the interlattice coupling strength $\gamma=\gamma_{u x}$, with $R_{x}=5, R_{u}=20$, and $\gamma_{x u}=0$. Other parameters: $\alpha=0.2, \beta=0.04, J=0.15$, $\delta=0.5, \varepsilon=0.005, \sigma_{x, u}=0.08$.

\section{Conclusions}

Effects of the mutual and external synchronization in the two coupled 2D ensembles of Nekorkin maps are studied in the work using methods of numerical experiments. We consider two cases of the interlayer coupling, diffusive and inertial, and compare the results. It is shown that there are no critical differences in synchronization effects for the coupling cases considered. There are only small differences in the synchronization thresholds and in the number of desynchronized oscillator pairs. There is some number of desynchronized oscillator pairs in all the 
cases considered. These important results indicate that both regular spiral waves and spiral waves with an incoherent core (spiral wave chimera states) do not realize the effects of complete synchronization even in different coupling cases. It is shown that there is a cluster of desynchronized oscillators not only when obvious incoherent cores are observed in the spiral wave chimera, but also when the regular spiral wave is realized.

\section{References}

[1] Pecora, L. M., Sorrentino, F., Hagerstrom, A. M., Murphy, T. E., and Roy, R., Cluster Synchronization and Isolated Desynchronization in Complex Networks with Symmetries, Nat. Commun., 2014, vol. 5, Art. 4079.

[2] Afraimovich, V.S., Nekorkin, V.I., Osipov, G.V., and Shalfeev, V.D., Stability, Structures and Chaos in Nonlinear Synchronization Network, World Sci. Ser. Nonlinear Sci. Ser. A Monogr. Treatises, vol. 6, Singapore: World Sci., 1994.

[3] Nekorkin, V.I., Kazantsev, V.B., and Velarde, M. G., Mutual Synchronization of Two Lattices of Bistable Elements, Phys. Lett. A, 1997, vol. 236, nos. 5-6, pp. 505-512.

[4] Nekorkin, V. I., Voronin, M. L., and Velarde, M. G., Clusters in an Ensemble of Globally Coupled Bistable Oscillators, Eur. Phys. J. B, 1999, vol. 9, no. 3, pp. 533-543.

[5] Belykh, V. N., Belykh, I. V., and Hasler, M., Hierarchy and Stability of Partially Synchronous Oscillations of Diffusively Coupled Dynamical Systems, Phys. Rev. E (3), 2000, vol. 62, no. 5, part A, pp. 6332-6345.

[6] Belykh, V.N., Belykh, I.V., and Mosekilde, E., Cluster Synchronization Modes in an Ensemble of Coupled Chaotic Oscillators, Phys. Rev. E, 2001, vol. 63, no. 3, 036216, 4 pp.

[7] Nekorkin, V.I., Kazantsev, V. B., and Velarde, M. G., Synchronization in Two-Layer Bistable Coupled Map Lattices, Phys. D, 2001, vol. 151, no. 1, pp.1-26.

[8] Nekorkin, V.I. and Velarde, M. G., Synergetic Phenomena in Active Lattices: Patterns, Waves, Solitons, Chaos, Berlin: Springer, 2002.

[9] Akopov, A., Astakhov, V., Vadivasova, T., Shabunin, A., and Kapitaniak, T., Frequency Synchronization of Clusters in Coupled Extended Systems, Phys. Lett. A, 2005, vol.334, nos. 2-3, pp. 169-172.

[10] Kuramoto, Y., Reduction Methods Applied to Nonlocally Coupled Oscillator Systems, in Nonlinear Dynamics and Chaos: Where Do We Go from Here?, J.Hogan et al. (Eds.), Boca Raton, Fla.: Chapman \& Hall/CRC, 2002, pp. 209-227.

[11] Kuramoto, Y. and Battogtokh, D., Coexistence of Coherence and Incoherence in Nonlocally Coupled Phase Oscillators, Nonlinear Phenom. Complex Syst., 2002, vol. 5, no. 4, pp. 380-385.

[12] Abrams, D. M. and Strogatz, S. H., Chimera States for Coupled Oscillators, Phys. Rev. Lett., 2004, vol. 93, no. 17, 174102, 4 pp.

[13] Panaggio, M. J. and Abrams, D. M., Chimera States: Coexistence of Coherence and Incoherence in Networks of Coupled Oscillators, Nonlinearity, 2015, vol. 28, no. 3, R67-R87.

[14] De Domenico, M., Solé-Ribalta, A., Cozzo, E., Kivelä, M., Moreno, Y., Porter, M. A., Gómez, S., and Arenas, A., Mathematical Formulation of Multilayer Networks, Phys. Rev. X, 2013, vol. 3, no. 4, 041022, 15 pp.

[15] Kivelä, M., Arenas, A., Barthelemy, M., Gleeson, J.P., Moreno, Y., and Porter, M. A., Multilayer Networks, J. Complex Netw., 2014, vol. 2, no. 3, pp. 203-271.

[16] Boccaletti, S., Bianconi, G., Criado, R., del Genio, C. I., Gómez-Gardeñes, J., Romance, M., SendiñaNadal, I., Wang, Z., and Zanin, M., The Structure and Dynamics of Multilayer Networks, Phys. Rep., 2014 , vol. 544 , no. 1 , pp. $1-122$. 
[17] Gambuzza, L. V., Frasca, M., and Gómez-Gardeñes, J., Intra-Layer Synchronization in Multiplex Networks, Europhys. Lett., 2015, vol.110, no. 2, 20010, 6 pp.

[18] Ghosh, S., Kumar, A., Zakharova, A., and Jalan, S., Birth and Death of Chimera: Interplay of Delay and Multiplexing, Europhys. Lett., 2016, vol.115, no. 6, 60005, 7 pp.

[19] Sevilla-Escoboza, R., Sendiña-Nadal, I., Leyva, I., Gutiérrez, R., Buldú, J. M., and Boccaletti, S., Inter-Layer Synchronization in Multiplex Networks of Identical Layers, Chaos, 2016, vol. 26, no.6, 065304, 6 pp.

[20] Ghosh, S. and Jalan, S., Emergence of Chimera in Multiplex Network, Internat. J. Bifur. Chaos Appl. Sci. Engrg., 2016, vol.26, no. 7, 1650120, 10 pp.

[21] Maksimenko, V.A., Makarov, V.V., Bera, B.K., Ghosh, D., Dana, S.K., Goremyko, M.V., Frolov, N. S., Koronovskii, A. A., and Hramov, A. E., Excitation and Suppression of Chimera States by Multiplexing, Phys. Rev. E, 2016, vol. 94, no. 5, 052205, 9 pp.

[22] Mikhaylenko, M., Ramlow, L., Jalan, S., and Zakharova, A., Weak Multiplexing in Neural Networks: Switching between Chimera and Solitary States, Chaos, 2019, vol. 29, no. 2, 023122, 10 pp.

[23] Andrzejak, R. G., Ruzzene, G., and Malvestio, I., Generalized Synchronization between Chimera States, Chaos, 2017, vol.27, no. 5, 053114, 6 pp.

[24] Singh, A., Ghosh, S., Jalan, S., and Kurths, J., Synchronization in Delayed Multiplex Networks, Europhys. Lett., 2015, vol. 111, no. 3, 30010, 6 pp.

[25] Boccaletti, S., Almendral, J.A., Guan, S., Leyva, I., Liu, Z., Sendiña-Nadal, I., Wang, Z., and Zou, Y., Explosive Transitions in Complex Networks-Structure and Dynamics: Percolation and Synchronization, Phys. Rep., 2016, vol.660, pp. 1-94.

[26] Bukh, A., Rybalova, E., Semenova, N., Strelkova, G., and Anishchenko, V., New Type of Chimera and Mutual Synchronization of Spatiotemporal Structures in Two Coupled Ensembles of Nonlocally Interacting Chaotic Maps, Chaos, 2017, vol.27, no. 11, 111102, 7 pp.

[27] Bukh, A. V., Strelkova, G. I., and Anishchenko, V. S., Synchronization of Chimera States in Coupled Networks of Nonlinear Chaotic Oscillators, Rus. J. Nonlin. Dyn., 2018, vol. 14, no. 4, pp. 419-433.

[28] Strelkova, G.I., Vadivasova, T.E., and Anishchenko, V.S., Synchronization of Chimera States in a Network of Many Unidirectionally Coupled Layers of Discrete Maps, Regul. Chaotic Dyn., 2018, vol. 23, nos. 7-8, pp. 948-960.

[29] Rybalova, E. V., Vadivasova, T. E., Strelkova, G. I., Anishchenko, V.S. and Zakharova, A. S., Forced Synchronization of a Multilayer Heterogeneous Network of Chaotic Maps in the Chimera State Mode, Chaos, 2019, vol. 29, no. 3, 033134, 9 pp.

[30] Leyva, I., Sevilla-Escoboza, R., Sendiña-Nadal, I., Gutiérrez, R., Buldú, J. M. and Boccaletti, S., Inter-Layer Synchronization in Non-Identical Multi-Layer Networks, Sci. Rep., 2017, vol.7, Art. 45475, 9 pp.

[31] Sawicki, J., Omelchenko, I., Zakharova, A., and Schöll, E., Delay Controls Chimera Relay Synchronization in Multiplex Networks, Phys. Rev. E, 2018, vol.98, no. 6, 062224, 9 pp.

[32] Zhang, X., Boccaletti, S., Guan, S., and Liu, Z., Explosive Synchronization in Adaptive and Multilayer Networks, Phys. Rev. Lett., 2015, vol. 114, no. 3, 038701, 5 pp.

[33] Leyva, I., Sendiña-Nadal, I., Sevilla-Escoboza, R., Vera-Avila, V. P., Chholak, P., and Boccaletti, S., Relay Synchronization in Multiplex Networks, Sci. Rep., 2018, vol.8, no. 1, Art.8629, 8 pp.

[34] Leyva, I., Sendiña-Nadal, I., and Boccaletti, S., Explosive Synchronization in Mono and Multilayer Networks, Discrete Contin. Dyn. Syst. Ser. B, 2018, vol. 23, no. 5, pp. 1931-1944.

[35] Kachhvah, A. D. and Jalan, S., Delay Regulated Explosive Synchronization in Multiplex Networks, New J. Phys., 2019, vol.21, no. 1, 015006, 14 pp.

[36] Omelchenko, I., Maistrenko, Yu., Hövel, P., and Schöll, E., Loss of Coherence in Dynamical Networks: Spatial Chaos and Chimera States, Phys. Rev. Lett., 2011, vol. 106, no. 23, 234102, 4 pp.

[37] Omelchenko, I., Riemenschneider, B., Hövel, Ph., and Schöll, E., Transition from Spatial Coherence to Incoherence in Coupled Chaotic Systems, Phys. Rev. E, 2012, vol. 85, no. 2, 026212, 9 pp. 
[38] Hagerstrom, A. M., Murphy, Th. E., Roy, R., Hövel, P., Omelchenko, I., and Schöll, E., Experimental Observation of Chimeras in Coupled-Map Lattices, Nature Phys., 2012, vol. 8, pp. 658-661.

[39] Tinsley, M. R., Nkomo, S., and Showalter, K., Chimera and Phase-Cluster States in Populations of Coupled Chemical Oscillators, Nature Phys., 2012, vol. 8, no. 9, pp. 662-665.

[40] Larger, L., Penkovsky, B., and Maistrenko, Yu., Virtual Chimera States for Delayed-Feedback Systems, Phys. Rev. Lett., 2013, vol. 111, no. 5, 054103, 5 pp.

[41] Martens, E. A., Thutupalli, S., Fourrière, A., and Hallatschek, O., Chimera States in Mechanical Oscillator Networks, Proc. Natl. Acad. Sci., 2013, vol.110, no. 26, pp. 10563-10567.

[42] Panaggio, M. J. and Abrams, D. M., Chimera States on a Flat Torus, Phys. Rev. Lett., 2013, vol. 110, no. 9, 094102, 5 pp.

[43] Dudkowski, D., Maistrenko, Yu., and Kapitaniak, T., Different Types of Chimera States: An Interplay between Spatial and Dynamical Chaos, Phys. Rev. E, 2014, vol. 90, no. 3, 032920, 5 pp.

[44] Maistrenko, Yu.L., Vasylenko, A., Sudakov, O., Levchenko, R., and Maistrenko, V.L., Cascades of Multiheaded Chimera States for Coupled Phase Oscillators, Internat. J. Bifur. Chaos Appl. Sci. Engrg., 2014, vol. 24, no. 8, 1440014, 17 pp.

[45] Yeldesbay, A., Pikovsky, A., and Rosenblum, M., Chimeralike States in an Ensemble of Globally Coupled Oscillators, Phys. Rev. Lett., 2014, vol. 112, no. 14, 144103, 5 pp.

[46] Kapitaniak, T., Kuzma, P., Wojewoda, J., Czolczynski, K., and Maistrenko, Yu., Imperfect Chimera States for Coupled Pendula, Sci. Rep., 2014, vol.4, Art. No.6379, 4 pp.

[47] Hizanidis, J., Panagakou, E., Omelchenko, I., Schöll, E., Hövel, Ph., and Provata, A., Chimera States in Population Dynamics: Networks with Fragmented and Hierarchical Connectivities, Phys. Rev. E, 2015, vol. 92, no. 1, 012915, 11 pp.

[48] Semenova, N., Zakharova, A., Schöll, E., and Anishchenko, V., Does Hyperbolicity Impede Emergence of Chimera States in Networks of Nonlocally Coupled Chaotic Oscillators?, Europhys. Lett., 2015, vol. 112, no. 4, 40002, 6 pp.

[49] Olmi, S., Martens, E. A., Thutupalli, S., and Torcini, A., Intermittent Chaotic Chimeras for Coupled Rotators, Phys. Rev. E, 2015, vol. 92, no. 3. 030901, 6 pp.

[50] Panaggio, M. J. and Abrams, D. M., Chimera States on the Surface of a Sphere, Phys. Rev. E (3), 2015, vol. 91, no. 2, 022909, 10 pp.

[51] Kemeth, F. P., Haugland, S. W., Schmidt, L., Kevrekidis, I. G., and Krischer, K., A Classification Scheme for Chimera States, Chaos, 2016, vol. 26, no. 9, 094815, 8 pp.

[52] Vadivasova, T.E., Strelkova, G. I., Bogomolov, S. A., and Anishchenko, V. S., Correlation Analysis of the Coherence-Incoherence Transition in a Ring of Nonlocally Coupled Logistic Maps, Chaos, 2016, vol. 26, no. 9, 093108, 9 pp.

[53] Schöll, E., Synchronization Patterns and Chimera States in Complex Networks: Interplay of Topology and Dynamics, Eur. Phys. J. Spec. Top., 2016, vol. 225, nos. 6-7, pp. 891-919.

[54] Ulonska, S., Omelchenko, I., Zakharova, A., and Schöll, E., Chimera States in Networks of van der Pol Oscillators with Hierarchical Connectivities, Chaos, 2016, vol. 26, no. 9, 094825, 9 pp.

[55] Semenova, N., Zakharova, A., Anishchenko, V., and Schöll, E., Coherence-Resonance Chimeras in a Network of Excitable Elements, Phys. Rev. Lett., 2016, vol. 117, no. 1, 014102, 6 pp.

[56] Semenov, V., Zakharova, A., Maistrenko, Yu., and Schöll, E., Delayed-Feedback Chimera States: Forced Multiclusters and Stochastic Resonance, Europhys. Lett., 2016, vol. 115, no. 1, 10005, 6 pp.

[57] Hizanidis, J., Kouvaris, N. E., Zamora-López, G., Díaz-Guilera, A., and Antonopoulos, C. G., Chimera-Like States in Modular Neural Networks, Sci. Rep., 2016, vol. 6, Art. No. 19845, 10 pp.

[58] Majhi, S., Perc, M., and Ghosh, D., Chimera States in Uncoupled Neurons Induced by a Multilayer Structure, Sci. Rep., 2016, vol. 6, Art. No. 39033, 10 pp.

[59] Sawicki, J., Omelchenko, I., Zakharova, A., and Schöll, E., Chimera States in Complex Networks: Interplay of Fractal Topology and Delay, Eur. Phys. J. Spec. Top., 2017, vol.226, no. 9, pp. 18831892. 
[60] Rybalova, E., Semenova, N., Strelkova, G., and Anishchenko, V., Transition from Complete Synchronization to Spatio-Temporal Chaos in Coupled Chaotic Systems with Nonhyperbolic and Hyperbolic Attractors, Eur. Phys. J. Spec. Top., 2017, vol. 226, no. 9, pp. 1857-1866.

[61] Bogomolov, S., Slepnev, A., Strelkova, G., Schöll, E., and Anishchenko, V., Mechanisms of Appearance of Amplitude and Phase Chimera States in Ensembles of Nonlocally Coupled Chaotic Systems, Commun. Nonlinear Sci. Numer. Simul., 2017, vol.43, pp. 25-36.

[62] Tian, Ch.-H., Zhang, X.-Y., Wang, Zh.-H., and Liu, Z.-H., Diversity of Chimera-Like Patterns from a Model of 2D Arrays of Neurons with Nonlocal Coupling, Front. Phys., 2017, vol. 12, no. 3, Art. 128904, 8 pp.

[63] Schmidt, A., Kasmatis, Th., Hizanidas, J., Provata, A., and Hövel, P., Chimera Patterns in TwoDimensional Networks of Coupled Neurons, Phys. Rev. E, 2017, vol. 95, no. 3, 032224, 13 pp.

[64] Shepelev, I. A., Bukh, A. V., Vadivasova, T. E., Anishchenko, V. S., and Zakharova, A., Double-Well Chimeras in 2D Lattice of Chaotic Bistable Elements, Commun. Nonlinear Sci. Numer. Simul., 2018, vol. 54, pp. 50-61.

[65] Hildebrand, M., Cui, J., Mihaliuk, E., Wang, J., and Showalter, K., Synchronization of Spatiotemporal Patterns in Locally Coupled Excitable Media, Phys. Rev. E (3), 2003, vol.68, no. 2, 026205, $4 \mathrm{pp}$.

[66] Kuramoto, Y. and Shima, Sh., Rotating Spirals without Phase Singularity in Reaction-Diffusion Systems. Let's Face Chaos through Nonlinear Dynamics (Maribor, 2002), Progr. Theoret. Phys. Suppl., 2003, No. 150, pp. 115-125.

[67] Shima, S. and Kuramoto, Y., Rotating Spiral Waves with Phase-Randomized Core in Nonlocally Coupled Oscillators, Phys. Rev. E, 2004, vol. 69, no. 3, 036213, 9 pp.

[68] Martens, E.A., Laing, C.R., and Strogatz, S.H., Solvable Model of Spiral Wave Chimeras, Phys. Rev. Lett., 2010, vol. 104, no. 4, 044101, 4 pp.

[69] Tang, X., Yang, T., Epstein, I. R., Liu, Y., Zhao, Y., and Gao, Q., Novel Type of Chimera Spiral Waves Arising from Decoupling of a Diffusible Component, J. Chem. Phys., 2014, vol.141, no. 2, 024110, $7 \mathrm{pp}$.

[70] Xie, J., Knobloch, E., and Kao, H.-C., Twisted Chimera States and Multicore Spiral Chimera States on a Two-Dimensional Torus, Phys. Rev. E, 2015, vol. 92, no. 4, 042921, 16 pp.

[71] Totz, J.F., Rode, J., Tinsley, M.R., Showalter, K., and Engel, H., Spiral Wave Chimera States in Large Populations of Coupled Chemical Oscillators, Nat. Phys., 2018, vol. 14, no. 3, pp. $282-285$.

[72] Laing, C. R., The Dynamics of Chimera States in Heterogeneous Kuramoto Networks, Phys. D, 2009, vol. 238, no. 16, pp. 1569-1588.

[73] Nkomo, S., Tinsley, M. R., and Showalter, K., Chimera States in Populations of Nonlocally Coupled Chemical Oscillators, Phys. Rev. Lett., 2013, vol. 110, no. 24, 244102, 5 pp.

[74] Gu, Ch., St-Yves, G., and Davidsen, J., Spiral Wave Chimeras in Complex Oscillatory and Chaotic Systems, Phys. Rev. Lett., 2013, vol. 111, no. 13, 134101, 5 pp.

[75] Kuramoto, Y., Shima, S., Battogtokh, D., and Shiogai, Y., Mean-Field Theory Revives in SelfOscillatory Fields with Non-Local Coupling, Prog. Theor. Phys. Suppl., 2006, vol. 161, pp. 127-143.

[76] Li, B.-W. and Dierckx, H., Spiral Wave Chimeras in Locally Coupled Oscillator Systems, Phys. Rev. E, 2016, vol.93, no. 2, 020202, 5 pp.

[77] Weiss, S. and Deegan, R. D., Weakly and Strongly Coupled Belousov-Zhabotinsky Patterns, Phys. Rev. E, 2017, vol. 95, no. 2, 022215, 11 pp.

[78] Kundu, S., Majhi, S., Muruganandam, P., and Ghosh, D., Diffusion Induced Spiral Wave Chimeras in Ecological System, Eur. Phys. J. Spec. Top., 2018, vol. 227, pp. 983-993.

[79] Guo, S., Dai, Q., Cheng, H., Li, H., Xie, F., and Yang, J., Spiral Wave Chimera in TwoDimensional Nonlocally Coupled Fitzhugh-Nagumo Systems, Chaos Solitons Fractals, 2018, vol. 114, pp. 394-399. 
[80] Bukh, A., Strelkova, G., and Anishchenko, V., Spiral Wave Patterns in a Two-Dimensional Lattice of Nonlocally Coupled Maps Modeling Neural Activity, Chaos Solitons Fractals, 2019, vol.120, pp. 75-82.

[81] Nekorkin, V.I. and Vdovin, L. V., Map-Based Model of the Neural Activity, Izv. Vyssh. Uchebn. Zaved. Prikl. Nelin. Dinam., 2007, vol.15, no. 5, pp. 36-60 (Russian).

[82] Bukh, A. V., Schöll, E., and Anishchenko, V.S., Synchronization of Spiral Wave Patterns in TwoLayer 2D Lattices of Nonlocally Coupled Discrete Oscillators, Chaos, 2019, vol. 29, no. 5, 053105, 7 pp.

[83] Bukh, A.V., Rybalova, E. V., and Anishchenko, V.S., Autowave Structures in Two-Dimensional Lattices of Nonlocally Coupled Oscillators, Izv. Vyssh. Uchebn. Zaved. Prikl. Nelin. Dinam., 2020, vol. 28 , no. 3 (in press).

[84] Pikovsky, A. and Politi, A., Lyapunov Exponents: A Tool to Explore Complex Dynamics, Cambridge: Cambridge Univ. Press, 2016. 